\title{
On beams made of a phase-transforming material
}

\author{
Prashant K. Purohit, Kaushik Bhattacharya * \\ Division of Engineering and Applied Science, California Institute of Technology, Mail Stop 104-44, Pasadena, CA 91125, USA
}

Received 13 February 2002

\begin{abstract}
This paper presents a theory for the mechanics of beams made of single crystals of shape memory alloys. The behavior of such beams can be quite unexpected and complicated due to the presence and propagation of phase boundaries. It is shown that the usual laws of mechanics do not fully determine the propagation of phase boundaries and that there is a need for additional constitutive information in the form of a kinetic relation. A simple experiment to measure this kinetic relation is proposed. Finally, a strategy to use such beams for propulsion at small scales is presented. (c) 2002 Elsevier Science Ltd. All rights reserved.
\end{abstract}

Keywords: Beams; Shape memory alloys; Martensitic transformation; Kinetic relation; Propulsion

\section{Introduction}

There are several technological applications of shape memory alloys including medical devices (such as stents, guide wires and dental arch-wires) and thermal actuators (such as thermostats and valves in IC engines and ventilation systems). In fact, the number of innovative products that exploit the unique properties of shape memory materials is steadily increasing. Most of these devices are constructed from Nickel-Titanium (NiTi) wire, strips or tubes and they rely on the flexural (as in strips and tubes), torsional (as in wire springs) and extensional (as in wires and tubes) characteristics of the alloy. Proper design of these devices requires an understanding of the behavior of these alloys in the form of lower dimensional structures. However, much of the current literature on shape memory alloys focusses on uniaxial extension of bars or wires following the landmark paper of Ericksen (1975) or full dimensional continua.

A notable exception is the work of Berg $(1994,1995)$ who studied bending of a polycrystalline wire of NiTi. In addition to traditional three and four point bending experiments, he designed and constructed an innovative device to subject these wires to a pure moment. He observed that phase transformation was characterized by a dramatic change in the curvature, and a hysteretic moment-curvature relation very similar to the commonly observed pseudoelastic force-extension curves. Motivated by these experiments, he proposed a beam theory with a non-monotone moment-curvature relation. In particular, his theory

\footnotetext{
${ }^{*}$ Corresponding author. Tel.: +1-626-395-8306; fax: +1-626-583-4963.

E-mail addresses: prashant@caltech.edu (P.K. Purohit), bhatta@caltech.edu (K. Bhattacharya).
} 
followed the Euler-Bernoulli ansatz that the centerline is inextensible and that the shear is zero (plane cross-sections remain plane normal to the center-line).

More recently, Bhattacharya and James (1999) have been motivated by experimental advances in thin films to derive directly a theory of thin films starting from a widely accepted three-dimensional theory. Bhattacharya (in press) has extended this procedure to rods. Their work is in a variational setting and applicable for films and rods made of single crystals of shape-memory alloys. They find that the leading term in the energy at small thickness is the energy associated with stretch and shear. Further, this energy inherits the multi-well structure from the three-dimensional energy. They further find that the energy of bending (and torsion) are corrections at higher order, and that the energy associated with these are quadratic and therefore do not have a multi-well character.

In this paper, we study the mechanics of beams made of a single crystal of a shape-memory alloy. Of particular interest is the laws that govern the propagation and evolution of phase boundaries. This issue has been widely studied in the one-dimensional extensional setting of bars (see for example, Abeyaratne and Knowles, 1991; Truskinovsky, 1987). It has been found that the usual balance laws do not completely determine the propagation velocity of phase boundaries, and that this has to be provided from outside the theory in the form of an additional constitutive relation known as the kinetic relation. While this theoretical framework has gained wide acceptance, it has proved to be difficult to measure it experimentally (see for example Escobar and Clifton (1993)). We find that the notion of kinetic relation is relevant even in beams, and propose a simple experiment to measure this kinetic relation.

The basic theory - the kinematics, the balance laws, dissipation inequality and the constitutive assumptions - are presented in detail in Section 2. We allow our beams to stretch, shear and bend, and assume following Bhattacharya (in press) that the energy is non-convex in stretch and shear, but convex in the moment. The kinematics which follow a one director or one Cosserat vector formulation and the balance laws are classical (we refer the reader to Antman (1995) for background and detailed bibliographic notes). The constitutive relations are not. Therefore a detailed discussion of the motivation and relation of the constitutive constants to crystallographically measurable quantities is given. Anticipating the role of phase boundaries, the treatment of the dissipation inequality is more general, and leads to a notion of driving force on interfaces.

The equilibria of such beams are studied in Section 3. We show that equilibria which involve phase boundaries are characterized by discontinuities in tangent as well sharp changes in curvature of the centerline of the beam. The role of the kinetic relation in quasistatic evolution of phase boundaries is discussed, and a simple experiment based on a cantilever to measure the kinetic relation is described. We turn to dynamics in Section 4, and show that the jump conditions do not uniquely determine the velocity of the phase boundaries and that there is indeed room for a kinetic relation.

It would be desirable to confirm the need for a kinetic relation through the solution of simple initialboundary value problems like Riemann or impact problems. For example, in bars and strings, Abeyaratne and Knowles (1991) and Purohit and Bhattacharya (in press) respectively have shown that these problems admit a one parameter family of solutions, and the kinetic relation provides a selection criterion. Unfortunately, even Riemann and impact problems are too complicated to solve explicitly in beams. In particular, in contrast to bars and strings the solutions are not piecewise constant in strain etc. since the coupling between the linear and angular momentum equations does not allow us to reduce them to wave equations. We have been able to identify some special boundary conditions in impact problems when this coupling vanishes, and one is able to write a one-parameter family of solutions (see Purohit (2001)). Unfortunately, these boundary conditions are physically difficult to achieve, and hence the calculations are not included here.

Note that our constitutive assumptions are quite different from those of Berg: his non-convexity is in the bending, while ours is in the stretch and shear. We show in Section 3 that our model reproduces some aspects of his experiments but not others. We suspect that this is due to fact that his experiments are in 
polycrystals, while our theory addresses single crystals. It would therefore be very interesting to repeat his experiments, and those suggested in Section 3 here in single crystal specimens. It remains an interesting and open question, whether non-convexity in shear and stretch in single crystals can give rise to a non-convexity in bending in a polycrystal made of multiple grains.

Section 5 examines if such beams can be used for propulsion in a viscous medium. Various microbes use flagella and cillia to propel themselves through fluids. They propagate bending waves through the flagellum, and the resulting resistance from the fluid results in the propulsion. We propose a similar mechanism by propagating a large number of phase boundaries through the beam.

\section{Basic equations}

\subsection{Kinematics}

Consider a beam of length $L$ and constant cross-sectional area $A$ in the reference configuration. Let $x$ be a typical point on the centerline $(0, L)$ of the reference configuration and $t$ denote an instant of time. We assume that the diameter of the cross-section is much smaller than the length of the beam. Therefore, we assume that during any deformation of the beam, planar cross-sections of the beam remain planar (but not necessarily normal to the centerline). We further assume in this paper that the deformation of the centerline is planar. Therefore we use a one-director Cosserat description where the deformation is described by two vector fields: $\mathbf{y}(x, t)$ which describes the deformation of the centerline of the beam and $\hat{\mathbf{b}}(x, t),|\hat{\mathbf{b}}|=1$ which describes the orientation of the cross-section.

Above, both $\mathbf{y}$ and $\hat{\mathbf{b}}$ are two-vectors. This is illustrated in Fig. 1. We note that the assumption $|\hat{\mathbf{b}}|=1$ does not mean that the cross-sectional area of the beam is assumed to be constant as it deforms. Instead, it is assumed that the cross-sectional area is completely determined by the axial stretch and shear and thus no independent kinematic variable is necessary to describe it. Since $\hat{\mathbf{b}}$ is a planar unit-vector, it can be described uniquely by the angle $\theta$ it makes with the horizontal:

$$
\hat{\mathbf{b}}(x, t)=\cos (\theta(x, t)) \hat{\mathbf{i}}+\sin (\theta(x, t)) \hat{\mathbf{j}} .
$$

We use the notations $\hat{\mathbf{b}}$ and $\theta$ interchangeably.

A super-posed dot denotes material time derivative, i.e., partial derivative with respect to $t$ holding $x$ fixed. Therefore the velocity of a particle on the center-line is $\dot{\mathbf{y}}(x, t)$ while the rate of rotation of the director is $\dot{\theta}(x, t)$. We denote the stretch and the tangent to the centerline respectively as

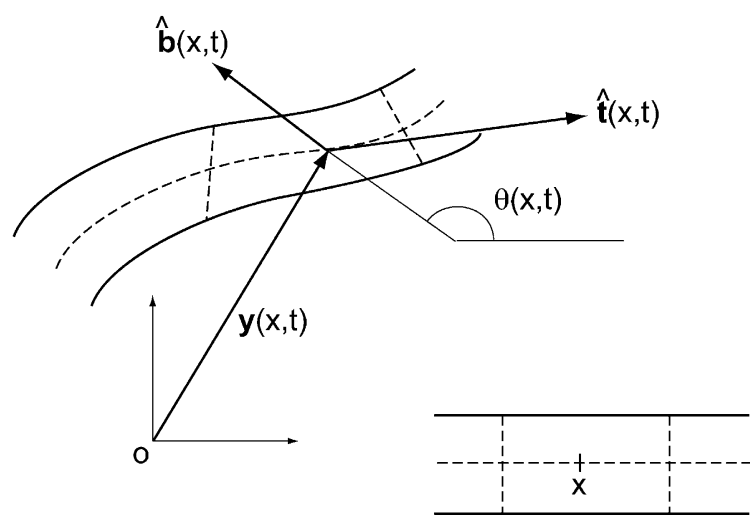

Fig. 1. Reference (bottom right) and deformed (top left) configuration of the beam. 


$$
\lambda=\left|\frac{\partial \mathbf{y}}{\partial x}\right|, \quad \hat{\mathbf{t}}=\frac{1}{\lambda} \frac{\partial \mathbf{y}}{\partial x} ; \quad \text { so } \frac{\partial \mathbf{y}}{\partial x}=\lambda \hat{\mathbf{t}} .
$$

It is useful to write this with respect to the orthonormal basis $\left\{\hat{\mathbf{b}}^{\perp}, \hat{\mathbf{b}}\right\}$ :

$$
\lambda \hat{\mathbf{t}}=\alpha \hat{\mathbf{b}}^{\perp}+\gamma \hat{\mathbf{b}} .
$$

Clearly $\lambda=\left(\alpha^{2}+\gamma^{2}\right)^{1 / 2}$ is the stretch and $\gamma$ is the shear. We also introduce the angle $\beta$ as the angle between $\hat{\mathbf{b}}$ and $\hat{\mathbf{t}}(\cos \beta=\hat{\mathbf{t}} \cdot \hat{\mathbf{b}})$. We have

$$
\alpha=\lambda \cos \beta, \quad \gamma=\lambda \sin \beta \text {. }
$$

Finally we define $\kappa=\partial \theta / \partial x$ to be the material or inherent curvature. We note that this may be different than the curvature of the centerline of the beam (which is equal to $\kappa / \lambda$ ) due to the stretch. Henceforth, we use curvature to mean material or inherent curvature unless specifically noted otherwise. The primary measures of strain in the beam are $\alpha, \gamma$ and $\kappa$. The reason for this choice will be clear when we study the balance laws and the dissipation inequality.

We are interested in studying shocks and phase boundaries. We therefore consider deformations $\mathbf{y}(x, t)$ and $\theta(x, t)$ that are not smooth, but only continuous in $x$ and $t$ with possible discontinuities in the velocities $\dot{\mathbf{y}}$ and $\dot{\theta}$, the stretch $\lambda$, the tangent $\hat{\mathbf{t}}$ and the curvature $\kappa$ at a finite number of points. Continuity of $\mathbf{y}(x, t)$ and $\theta(x, t)$ implies that these jumps cannot be arbitrary but satisfy some jump conditions. If we have discontinuities in the quantities listed above at the point $s(t)$ in the reference configuration, then

$$
\begin{aligned}
& -\dot{s}[|\lambda \hat{\mathbf{t}}|]=[|\dot{\mathbf{y}}|], \\
& -\dot{s}[|\kappa|]=[|\dot{\theta}|],
\end{aligned}
$$

where $\dot{s}$ is the velocity of the discontinuity in the reference configuration.

\subsection{Conservation laws}

The balance of linear momentum for a part of the beam occupying the interval $\left(x_{1}, x_{2}\right)$ requires

$$
\frac{\mathrm{d}}{\mathrm{d} t} \int_{x_{1}}^{x_{2}} \rho A \dot{\mathbf{y}} \mathrm{d} x=\mathbf{T}\left(x_{2}, t\right)-\mathbf{T}\left(x_{1}, t\right)+\int_{x_{1}}^{x_{2}} \mathbf{f} \mathrm{d} x,
$$

where $\rho$ is the density of the beam in the reference configuration, $A$ is its cross-sectional area in the reference configuration, $\mathbf{T}(x, t)$ is the force acting at material point $x$ at time $t$, and $\mathbf{f}(x)$ is the body force per unit reference length at material point $x$.

For future use, we denote $T_{\perp}=\mathbf{T} \cdot \hat{\mathbf{b}}^{\perp}$ and $T_{b}=\mathbf{T} \cdot \hat{\mathbf{b}}$ to be the components of $\mathbf{T}$ with respect to the orthonormal basis $\left\{\hat{\mathbf{b}}^{\perp}, \hat{\mathbf{b}}\right\}$.

If there are no discontinuities in the interval $\left(x_{1}, x_{2}\right)$ then (3) can be localized to

$$
\rho A \ddot{\mathbf{y}}=\frac{\partial \mathbf{T}}{\partial x}+\mathbf{f} .
$$

If there is a discontinuity in the interval $\left(x_{1}, x_{2}\right)$ then we also need a jump condition. If we have a discontinuity at $x=s(t)$ in the reference configuration then the required jump condition may be easily derived by dividing each integral in (3) into two: one from $x_{1}$ to $s(t)$ and the other from $s(t)$ to $x_{2}$. We obtain,

$$
-\dot{s}[|\rho A \dot{\mathbf{y}}|]=[|\mathbf{T}|] \text {. }
$$

We assume henceforth that $\rho$ and $A$ are constant and therefore we conclude that

$$
-\dot{s} \rho A[|\dot{\mathbf{y}}|]=[|\mathbf{T}|] .
$$


Similarly, the balance of angular momentum for the part of the beam in the interval $\left(x_{1}, x_{2}\right)$ requires

$$
\begin{aligned}
\frac{\mathrm{d}}{\mathrm{d} t} \int_{x_{1}}^{x_{2}} \mathbf{y} \times \rho A \dot{\mathbf{y}} \mathrm{d} x+\frac{\mathrm{d}}{\mathrm{d} t} \int_{x_{1}}^{x_{2}} \hat{\mathbf{b}} \times \rho I \dot{\hat{\mathbf{b}}} \mathrm{d} x= & M\left(x_{2}, t\right)-M\left(x_{1}, t\right)+\mathbf{y}\left(x_{2}, t\right) \times \mathbf{T}\left(x_{2}, t\right)-\mathbf{y}\left(x_{1}, t\right) \times \mathbf{T}\left(x_{1}, t\right) \\
& +\int_{x_{1}}^{x_{2}} \mathbf{y} \times \mathbf{f} \mathrm{d} x+\int_{x_{1}}^{x_{2}} l \mathrm{~d} x,
\end{aligned}
$$

where $I$ is the second moment of area of the cross-section of the beam about an axis that causes the first moment to vanish, $M(x, t)$ is the moment acting at material point $x$ at time $t$, and $l(x)$ is the body moment per unit reference length of the beam at material point $x$.

Note in the above expression that we write the cross-product as a scalar since we are working in two dimensions. We use this convention through out this paper. The statement above can be localized in the absence of discontinuities to

$$
\mathbf{y} \times \rho A \ddot{\mathbf{y}}+\hat{\mathbf{b}} \times \rho / \ddot{\hat{\mathbf{b}}}=\frac{\partial M}{\partial x}+\frac{\partial}{\partial x}(\mathbf{y} \times \mathbf{T})+\mathbf{y} \times \mathbf{f}+l .
$$

Recalling the definition of $\theta$ and using (4), this can be simplified to obtain

$$
\rho I \ddot{\theta}=\frac{\partial M}{\partial x}+\frac{\partial \mathbf{y}}{\partial x} \times \mathbf{T}+l
$$

or

$$
\rho I \ddot{\theta}=\frac{\partial M}{\partial x}+\lambda \hat{\mathbf{t}} \times \mathbf{T}+l .
$$

When we have a discontinuity at $x=s(t)$, we also obtain the following jump condition,

$$
-\dot{s} \rho I[|\dot{\theta}|]=[|M|] \text {. }
$$

In summary, the Eqs. (4) and (6) along with the jump conditions (5) and (7) describe the balance of linear and angular momentum in the beam.

We now turn to the dissipation inequality. Since we are considering a purely mechanical theory, we cannot write a balance of energy, but can write a dissipation inequality: rate of work being done by external forces (and moments) on any part of the body is greater than or equal to the rate of change of kinetic and potential energy in the same part of the body. Consider a portion of the beam in the interval $\left(x_{1}, x_{2}\right)$. The rate of work being done by external forces (and moments) on this part of the body is

$$
\begin{aligned}
P^{\mathrm{ext}}= & \int_{x_{1}}^{x_{2}}\{\dot{\mathbf{y}} \cdot \mathbf{f}+l(\hat{\mathbf{b}} \times \dot{\hat{\mathbf{b}}})\} \mathrm{d} x+\dot{\mathbf{y}}\left(x_{2}, t\right) \cdot \mathbf{T}\left(x_{2}, t\right)-\dot{\mathbf{y}}\left(x_{1}, t\right) \cdot \mathbf{T}\left(x_{1}, t\right)+M\left(x_{2}, t\right) \hat{\mathbf{b}}\left(x_{2}, t\right) \times \dot{\hat{\mathbf{b}}}\left(x_{2}, t\right) \\
& -M\left(x_{1}, t\right) \hat{\mathbf{b}}\left(x_{1}, t\right) \times \dot{\hat{\mathbf{b}}}\left(x_{1}, t\right) .
\end{aligned}
$$

Similarly, the rate of change of kinetic and potential energy in the same part of the body is given by

$$
P^{\text {int }}=\frac{\mathrm{d}}{\mathrm{d} t} \int_{x_{1}}^{x_{2}}\left\{\frac{\rho}{2}|\dot{\mathbf{y}}|^{2}+\frac{\rho I}{2}|\hat{\mathbf{b}} \times \dot{\hat{\mathbf{b}}}|^{2}+\Phi\right\} \mathrm{d} x
$$

where $\Phi=\Phi(x, t)$ is the energy stored in the beam per unit reference length. We define the power dissipated as

$$
P^{\text {diss }}=P^{\text {ext }}-P^{\text {int }} .
$$

The dissipation inequality states that

$$
P^{\text {diss }} \geqslant 0 \text { or } P^{\text {ext }} \geqslant P^{\text {int }} \text {. }
$$


For smooth motions,

$$
P^{\text {int }}=\int_{x_{1}}^{x_{2}}\{\dot{\mathbf{y}} \cdot \rho \ddot{\mathbf{y}}+(\hat{\mathbf{b}} \times \dot{\hat{\mathbf{b}}})(\hat{\mathbf{b}} \times \rho I \ddot{\hat{\mathbf{b}}})+\dot{\Phi}\} \mathrm{d} x .
$$

Therefore

$$
\begin{aligned}
P^{\mathrm{diss}}= & \int_{x_{1}}^{x_{2}}\{\dot{\mathbf{y}} \cdot(\mathbf{f}-\rho \ddot{\mathbf{y}})+(\hat{\mathbf{b}} \times \dot{\hat{\mathbf{b}}})(l-\hat{\mathbf{b}} \times \rho I \ddot{\hat{\mathbf{b}}})-\dot{\Phi}\} \mathrm{d} x+\dot{\mathbf{y}}\left(x_{2}, t\right) \cdot \mathbf{T}\left(x_{2}, t\right)-\dot{\mathbf{y}}\left(x_{1}, t\right) \cdot \mathbf{T}\left(x_{1}, t\right) \\
& +M\left(x_{2}, t\right) \hat{\mathbf{b}}\left(x_{2}, t\right) \times \dot{\hat{\mathbf{b}}}\left(x_{2}, t\right)-M\left(x_{1}, t\right) \hat{\mathbf{b}}\left(x_{1}, t\right) \times \dot{\hat{\mathbf{b}}}\left(x_{1}, t\right) .
\end{aligned}
$$

Using the balance of momenta, (4) and (6), this can be rewritten as

$$
\begin{aligned}
P^{\mathrm{diss}}= & -\int_{x_{1}}^{x_{2}}\left\{\frac{\partial \mathbf{T}}{\partial x} \cdot \dot{\mathbf{y}}+\left(\frac{\partial M}{\partial x}+\lambda \hat{\mathbf{t}} \times \mathbf{T}\right)(\hat{\mathbf{b}} \times \dot{\hat{\mathbf{b}}})+\dot{\Phi}\right\} \mathrm{d} x+\dot{\mathbf{y}}\left(x_{2}, t\right) \cdot \mathbf{T}\left(x_{2}, t\right)-\dot{\mathbf{y}}\left(x_{1}, t\right) \cdot \mathbf{T}\left(x_{1}, t\right) \\
& +M\left(x_{2}, t\right) \hat{\mathbf{b}}\left(x_{2}, t\right) \times \dot{\hat{\mathbf{b}}}\left(x_{2}, t\right)-M\left(x_{1}, t\right) \hat{\mathbf{b}}\left(x_{1}, t\right) \times \dot{\hat{\mathbf{b}}}\left(x_{1}, t\right) .
\end{aligned}
$$

We can now integrate by parts to obtain

$$
P^{\mathrm{diss}}=\int_{x_{1}}^{x_{2}}\left\{\mathbf{T} \cdot \frac{\partial \dot{\mathbf{y}}}{\partial x}+M \frac{\partial}{\partial x}(\hat{\mathbf{b}} \times \dot{\hat{\mathbf{b}}})-(\lambda \hat{\mathbf{t}} \times \mathbf{T})(\hat{\mathbf{b}} \times \dot{\hat{\mathbf{b}}})-\dot{\Phi}\right\} \mathrm{d} x
$$

or

$$
P^{\mathrm{diss}}=\int_{x_{1}}^{x_{2}}\left\{\mathbf{T} \cdot\left(\frac{\partial \dot{\mathbf{y}}}{\partial x}-(\hat{\mathbf{b}} \times \dot{\hat{\mathbf{b}}}) \lambda \hat{\mathbf{t}}^{\perp}\right)+M \frac{\partial}{\partial x}(\hat{\mathbf{b}} \times \dot{\hat{\mathbf{b}}})-\dot{\Phi}\right\} \mathrm{d} x
$$

since $\mathbf{T} \times \lambda \hat{\mathbf{t}}=-\mathbf{T} \cdot \lambda \hat{\mathbf{t}}^{\perp}$ for $\lambda \hat{\mathbf{t}}^{\perp}=-\gamma \hat{\mathbf{b}}^{\perp}+\alpha \hat{\mathbf{b}}$. Further, since $\hat{\mathbf{b}} \times \dot{\hat{\mathbf{b}}}=\dot{\theta}$, the above expression can be rewritten as

$$
P^{\mathrm{diss}}=\int_{x_{1}}^{x_{2}}\left\{\mathbf{T} \cdot\left(\frac{\partial \dot{\mathbf{y}}}{\partial x}-\dot{\theta} \lambda \hat{\mathbf{t}}^{\perp}\right)+M \dot{\kappa}-\dot{\Phi}\right\} \mathrm{d} x .
$$

We now show that

$$
\frac{\partial \dot{\mathbf{y}}}{\partial x}-\dot{\theta} \lambda \hat{\mathbf{t}}^{\perp}=\dot{\gamma} \hat{\mathbf{b}}+\dot{\alpha} \hat{\mathbf{b}}^{\perp}
$$

First,

$$
\dot{\gamma}=\frac{\dot{\lambda}}{\lambda \hat{\mathbf{t}} \cdot \hat{\mathbf{b}}}=\dot{\overline{\lambda \hat{\mathbf{t}}}} \cdot \hat{\mathbf{b}}+\lambda \hat{\mathbf{t}} \cdot \dot{\hat{\mathbf{b}}}=\dot{\bar{\lambda}} \cdot \hat{\mathbf{b}}^{\perp}-\dot{\theta} \lambda \hat{\mathbf{t}} \cdot \hat{\mathbf{b}}^{\perp}=\dot{\overline{\lambda \hat{\mathbf{t}}}} \cdot \hat{\mathbf{b}}^{\perp}-\dot{\theta} \alpha .
$$

Similarly,

$$
\dot{\alpha}=\dot{\overline{\lambda \hat{\mathbf{t}}}} \cdot \hat{\mathbf{b}}+\dot{\theta} \gamma \text {. }
$$

Therefore,

$$
\left.\dot{\gamma} \hat{\mathbf{b}}+\dot{\alpha} \hat{\mathbf{b}}^{\perp}=(\dot{\overline{\lambda \hat{\mathbf{t}}}} \cdot \hat{\mathbf{b}}) \hat{\mathbf{b}}+\dot{(\dot{\lambda \hat{\mathbf{t}}}} \cdot \hat{\mathbf{b}}^{\perp}\right) \hat{\mathbf{b}}^{\perp}-\dot{\theta}(\alpha \hat{\mathbf{b}}-\gamma \hat{\mathbf{b}})=\dot{\dot{\lambda \overrightarrow{\mathbf{t}}}}-\dot{\theta}(\alpha \hat{\mathbf{b}}-\gamma \hat{\mathbf{b}})
$$

which is (13). 
Using (13), we can rewrite (12) as

$$
P^{\text {diss }}=\int_{x_{1}}^{x_{2}}\left\{T_{b} \dot{\gamma}+T_{\perp} \dot{\alpha}+M \dot{\kappa}-\dot{\Phi}\right\} \mathrm{d} x .
$$

Therefore the dissipation inequality for smooth motions after localization says

$$
T_{b} \dot{\gamma}+T_{\perp} \dot{\alpha}+M \dot{\kappa}-\dot{\Phi} \geqslant 0
$$

We now consider a deformation with a discontinuity at $s(t)$. Starting from (11), we obtain the following condition in addition to (15).

$$
-\dot{S}[|\Phi|]-\rho A \dot{s}[|\dot{\mathbf{y}}|] \cdot\langle\dot{\mathbf{y}}\rangle-\rho I \dot{s}[|\dot{\theta}|]\langle\dot{\theta}\rangle \geqslant[|\mathbf{T}|] \cdot\langle\dot{\mathbf{y}}\rangle+\langle\mathbf{T}\rangle \cdot[|\dot{\mathbf{y}}|]+[|M|]\langle\dot{\theta}\rangle+\langle M\rangle[|\dot{\theta}|] .
$$

Using the jump conditions (5) and (7) we get

$$
-\dot{s}[|\Phi|] \geqslant\langle\mathbf{T}\rangle \cdot[|\dot{\mathbf{y}}|]+\langle M\rangle[|\dot{\theta}|]
$$

Finally using the kinematic conditions (1) and (2) we get

$$
\dot{s}([|\Phi|]-\langle\mathbf{T}\rangle \cdot[|\lambda \hat{\mathbf{t}}|]-\langle M\rangle[|\kappa|]) \geqslant 0 .
$$

We thus notice that the term in parenthesis is the force conjugate to the velocity of the discontinuity. Therefore, following Abeyaratne and Knowles (1990) and Eshelby $(1956,1975)$ we define it to be the thermodynamic driving force:

$$
f=[|\Phi|]-\langle\mathbf{T}\rangle \cdot[|\lambda \hat{\mathbf{t}}|]-\langle M\rangle[|\kappa|] .
$$

Therefore, the dissipation inequality becomes:

$$
f \dot{s} \geqslant 0 \text {. }
$$

\subsection{Constitutive assumptions}

We now make the following constitutitve assumptions.

$$
\begin{aligned}
& \Phi=\Phi(\alpha, \gamma, \kappa) \\
& \mathbf{T}=\mathbf{T}(\alpha, \gamma, \kappa) \\
& M=M(\alpha, \gamma, \kappa)
\end{aligned}
$$

Substituting these into (15), we write it as

$$
\left(T_{\perp}-\frac{\partial \Phi}{\partial \alpha}\right) \dot{\alpha}+\left(T_{b}-\frac{\partial \Phi}{\partial \gamma}\right) \dot{\gamma}+\left(M-\frac{\partial \Phi}{\partial \kappa}\right) \dot{\kappa} \geqslant 0 .
$$

Following Coleman and Noll (1963), we can argue that this inequality has to be true for all smooth motions (since we can arbitrarily choose the body forces and moments). In particular, we can choose a class of motions where $(\gamma, \alpha, \kappa)$ are the same, but $(\dot{\gamma}, \dot{\alpha}, \dot{\kappa})$ take arbitrary values. The above inequality has to hold for each of these motions. Therefore we conclude that

$$
T_{\perp}=\frac{\partial \Phi}{\partial \alpha}, \quad T_{b}=\frac{\partial \Phi}{\partial \gamma}, \quad M=\frac{\partial \Phi}{\partial \kappa} .
$$

Therefore, we only need to specify a constitutive relation for $\Phi$. We note that this also implies that the dissipation is zero for any smooth motion, and that the only possible source of dissipation in these beams is at the discontinuities. 
We now specialize to a specific constitutive relation, appropriate for beams made of single crystals of materials undergoing martensitic phase transformation. We will argue shortly that it is appropriate in such situations to assume that the energy is a non-convex function of the stretch and the shear, but a convex function of the curvature. We assume that there are two natural or stress-free states for the beam:

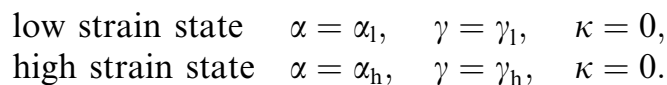

Above, $\alpha_{1}$ and $\alpha_{\mathrm{h}}$ are the axial transformation stretches, stress-free stretches or eigen-stretches in the two phases and $\gamma_{1}$ and $\gamma_{\mathrm{h}}$ are the transformation strains, stress-free strains or eigen-strains in shear. We assume that the moment-free curvature of both states is zero. The free energy $\Phi$ has local minima at these states and grows away from it. In order to keep things simple, we assume that $\Phi$ is quadratic near these states. We assume therefore that

$$
\Phi= \begin{cases}\frac{1}{2} E A\left(\alpha-\alpha_{1}\right)^{2}+\frac{1}{2} \mu A\left(\gamma-\gamma_{1}\right)^{2}+\frac{1}{2} E I \kappa^{2} & \text { in the low strain phase, } \\ \Phi_{\mathrm{u}}(\gamma, \alpha)+\frac{1}{2} E I \kappa^{2} & \text { in the unstable phase } \\ \frac{1}{2} E A\left(\alpha-\alpha_{\mathrm{h}}\right)^{2}+\frac{1}{2} \mu A\left(\gamma-\gamma_{\mathrm{h}}\right)^{2}+\frac{1}{2} E I \kappa^{2}+\Phi_{0} & \text { in the high strain phase }\end{cases}
$$

for a suitable $\Phi_{\mathrm{u}}$. Above, $E$ is the Young's modulus, $\mu$ the shear modulus and $\Phi_{0}$ the difference in the ground state energies of the two phases. Note that we have assumed that both phases have the same moduli. This is not true in actual materials, but it simplifies many subsequent calculations. $\Phi_{0}$ determines which phase would be more stable in a completely stress-free situation. We have not explicitly specified the regions of validity of the expressions for the low strain phase and high strain phase, neither have we given any explicit expression for the unstable phase $\Phi_{\mathrm{u}}$. We note that it is possible to specify these in such a manner that $\Phi$ is smooth and has no additional local minima. The details are cumbersome and omitted.

The expressions for the force $\mathbf{T}$ and the moment $M$ follow immediately from (20). Omitting the expressions in the unstable phase, we can write

$$
\mathbf{T}=E A\left(\lambda \hat{\mathbf{t}} \cdot \hat{\mathbf{b}}^{\perp}-\alpha^{*}\right) \hat{\mathbf{b}}^{\perp}+\mu A\left(\lambda \hat{\mathbf{t}} \cdot \hat{\mathbf{b}}-\gamma^{*}\right) \hat{\mathbf{b}}
$$

where

$$
\begin{aligned}
& \alpha^{*}= \begin{cases}\alpha_{1} & \text { low strain phase } \\
\alpha_{\mathrm{h}} & \text { high strain phase }\end{cases} \\
& \gamma^{*}= \begin{cases}\gamma_{1} & \text { low strain phase } \\
\gamma_{\mathrm{h}} & \text { high strain phase }\end{cases}
\end{aligned}
$$

and

$$
M=E I \kappa .
$$

We now discuss the justification for choosing an energy that is non-convex in stretch and shear but convex in the curvature. This follows from the work of Bhattacharya (in press), where a theory for rods made of martensitic material has been derived starting from a fully three-dimensional theory without the use of any a priori ansatz about the deformation. This derivation is in a variational setting appropriate for the study of equilibria, and follows the methods of Bhattacharya and James (1999).

In martensitic materials, we have a high temperature austenite phase, and a low temperature martensite phase. Typically, the symmetry of the austenite is greater than that of the martensite and this gives rise to multiple variants of martensite. There is now a well-developed continuum framework for modeling such materials (see for example, Ball and James (1992), Bhattacharya (1991)), where one describes different configurations of the crystal as deformations of some fixed reference configuration. It is conventional to 
choose the austenite phase as the reference, so the identity corresponds to the the austenite. Each variant of martensite can be obtained by an affine deformation of the austenite, and therefore we can describe the variants through fixed transformation stretch $\mathbf{U}_{j}$ which takes the austenite lattice to the martensite lattice. We assume that these are positive-definite and symmetric.

For example, there are three variants of martensite in a material undergoing cubic to tetragonal transformation, with

$$
\mathbf{U}_{1}=\left(\begin{array}{lll}
\eta_{2} & 0 & 0 \\
0 & \eta_{1} & 0 \\
0 & 0 & \eta_{1}
\end{array}\right), \quad \mathbf{U}_{2}=\left(\begin{array}{lll}
\eta_{1} & 0 & 0 \\
0 & \eta_{2} & 0 \\
0 & 0 & \eta_{1}
\end{array}\right), \quad \mathbf{U}_{3}=\left(\begin{array}{lll}
\eta_{1} & 0 & 0 \\
0 & \eta_{1} & 0 \\
0 & 0 & \eta_{2}
\end{array}\right),
$$

where the measured values in NiAl are $\eta_{1}=0.9392, \eta_{2}=1.1302$. Similarly there are six variants of martensite in a material undergoing cubic to orthorhombic transformation, with

$$
\mathbf{U}_{1}=\left(\begin{array}{ccc}
\frac{\eta_{1}+\eta_{3}}{2} & \frac{\eta_{1}-\eta_{3}}{2} & 0 \\
\frac{\eta_{1}-\eta_{3}}{2} & \frac{\eta_{1}+\eta_{3}}{2} & 0 \\
0 & 0 & \eta_{2}
\end{array}\right), \quad \mathbf{U}_{2}=\left(\begin{array}{ccc}
\frac{\eta_{1}+\eta_{3}}{2} & \frac{\eta_{3}-\eta_{1}}{2} & 0 \\
\frac{\eta_{3} \eta_{1}}{2} & \frac{\eta_{1}+\eta_{3}}{2} & 0 \\
0 & 0 & \eta_{2}
\end{array}\right)
$$

and the rest obtained by symmetry where the measured values in CuAlNi are $\eta_{1}=1.0619, \eta_{2}=0.9178$, $\eta_{3}=1.0231$.

Suppose we make a beam of the material in the austenite phase, and pick an orthonormal basis $\left\{\hat{\mathbf{e}}_{1}, \hat{\mathbf{e}}_{2}, \hat{\mathbf{e}}_{3}\right\}$ with $\hat{\mathbf{e}}_{1}$ the axis of the beam and $\hat{\mathbf{e}}_{2}$ the transverse direction. Now suppose the beam goes through a phase transformation (say due to cooling) and transforms completely to a variant whose stress-free configuration is described by an affine deformation with gradient $\mathbf{U}_{i}$. The directions $\left\{\hat{\mathbf{e}}_{i}\right\}$ now are deformed to $\left\{\mathbf{U}_{j} \hat{\mathbf{e}}_{i}\right\}$. Suppose further that $\mathbf{U}_{j}$ and the directions $\left\{\hat{\mathbf{e}}_{i}\right\}$ were chosen such that this deformation is planar except for a uniform stretch in the $\hat{\mathbf{e}}_{3}$ direction, i.e.,

$$
\hat{\mathbf{e}}_{1} \cdot \mathbf{U}_{j}^{2} \hat{\mathbf{e}}_{3}=\hat{\mathbf{e}}_{2} \cdot \mathbf{U}_{j}^{2} \hat{\mathbf{e}}_{3}=0 .
$$

We can treat this now as a beam in the $\mathbf{U}_{j} \hat{\mathbf{e}}_{1}-\mathbf{U}_{j} \hat{\mathbf{e}}_{2}$ plane. The stress-free configuration is described by

$$
\lambda=\left|\mathbf{U}_{j} \hat{\mathbf{e}}_{1}\right|, \quad \beta=\arccos \left(\frac{\hat{\mathbf{e}}_{2} \cdot \mathbf{U}_{j}^{2} \hat{\mathbf{e}}_{1}}{\left|\mathbf{U}_{j} \hat{\mathbf{e}}_{1}\right|\left|\mathbf{U}_{j} \hat{\mathbf{e}}_{2}\right|}\right)
$$

or equivalently

$$
\alpha=\frac{1}{\left|\mathbf{U}_{j}^{-\mathrm{T}} \hat{\mathbf{e}}_{1}\right|}, \quad \gamma=\frac{\hat{\mathbf{e}}_{2} \cdot \mathbf{U}_{j}^{2} \hat{\mathbf{e}}_{1}}{\left|\mathbf{U}_{j} \hat{\mathbf{e}}_{2}\right|} .
$$

Note that rigid body rotations of this beam $\mathbf{U}_{j} \rightarrow \mathbf{Q \mathbf { U } _ { j }}$ do not change these quantities. Finally the momentfree curvature of this beam is zero.

Suppose we have a beam where only the austenite and the first variant of martensite are active. Then, we have to choose $\left\{\hat{\mathbf{e}}_{i}\right\}$ such that (24) holds for $j=1$. We now have two phases with

$$
\alpha_{1}=1, \quad \alpha_{\mathrm{h}}=\frac{1}{\left|\mathbf{U}_{1}^{-\mathrm{T}} \hat{\mathbf{e}}_{1}\right|}, \quad \gamma_{1}=0, \quad \gamma_{\mathrm{h}}=\frac{\hat{\mathbf{e}}_{2} \cdot \mathbf{U}_{1}^{2} \hat{\mathbf{e}}_{1}}{\left|\mathbf{U}_{1} \hat{\mathbf{e}}_{2}\right|}
$$

and $\Phi_{0}$ depends on temperature. Similarly, if we have a beam where only the first two variants are active. Then, we have to choose $\left\{\hat{\mathbf{e}}_{i}\right\}$ such that (24) holds for both $j=1$ and $j=2$. We now have two phases with

$$
\alpha_{1}=\frac{1}{\left|\mathbf{U}_{1}^{-\mathrm{T}} \hat{\mathbf{e}}_{1}\right|}, \quad \alpha_{\mathrm{h}}=\frac{1}{\left|\mathbf{U}_{2}^{-\mathrm{T}} \hat{\mathbf{e}}_{2}\right|}, \quad \gamma_{1}=\frac{\hat{\mathbf{e}}_{2} \cdot \mathbf{U}_{1}^{2} \hat{\mathbf{e}}_{1}}{\left|\mathbf{U}_{1} \hat{\mathbf{e}}_{2}\right|}, \quad \gamma_{\mathrm{h}}=\frac{\hat{\mathbf{e}}_{2} \cdot \mathbf{U}_{2}^{2} \hat{\mathbf{e}}_{1}}{\left|\mathbf{U}_{2} \hat{\mathbf{e}}_{2}\right|}
$$


and $\Phi_{0}$ is zero. In either case, we have two distinct values for the stress-free values of $\alpha$ and $\gamma$. At the same time, the moment-free curvature is zero in each of these states. Therefore, it is natural to assume nonconvexity of the energy in stretch and shear, but convexity in curvature.

We conclude by noting that even in one given material, one can freely change the stress-free values of $\alpha$ and $\gamma$ by simply changing the crystallographic orientation of the beam $\left\{\hat{\mathbf{e}}_{i}\right\}$. Consider for example the material undergoing cubic to orthorhombic transformation as in CuAlNi. Note that the following choice of $\left\{\hat{\mathbf{e}}_{i}\right\}$ satisfies (24) for both variants 1 and 2:

$$
\hat{\mathbf{e}}_{1}=\left(\begin{array}{c}
\cos \xi \\
\sin \xi \\
0
\end{array}\right), \quad \hat{\mathbf{e}}_{2}=\left(\begin{array}{c}
-\sin \xi \\
\cos \xi \\
0
\end{array}\right), \quad \hat{\mathbf{e}}_{3}=\left(\begin{array}{l}
0 \\
0 \\
1
\end{array}\right) .
$$

It is easy to verify that

$$
\alpha_{1}=\frac{\sqrt{2} \eta_{1} \eta_{3}}{\sqrt{\eta_{1}^{2}+\eta_{3}^{2}+2 \sin \xi \cos \xi\left(\eta_{1}^{2}-\eta_{3}^{2}\right)}}, \quad \alpha_{\mathrm{h}}=\frac{\sqrt{2} \eta_{1} \eta_{3}}{\sqrt{\eta_{1}^{2}+\eta_{3}^{2}+2 \sin \xi \cos \xi\left(\eta_{3}^{2}-\eta_{1}^{2}\right)}},
$$

and

$$
\gamma_{1}=\frac{4 \sqrt{2} \eta_{3}^{2} \sin \xi \cos \xi}{\sqrt{\eta_{1}^{2}+\eta_{3}^{2}+2\left(\eta_{1}^{2}-\eta_{3}^{2}\right) \sin \xi \cos \xi}}, \quad \gamma_{\mathrm{h}}=-\frac{4 \sqrt{2} \eta_{1}^{2} \sin \xi \cos \xi}{\sqrt{\eta_{1}^{2}+\eta_{3}^{2}+2\left(\eta_{3}^{2}-\eta_{1}^{2}\right) \sin \xi \cos \xi}} .
$$

\subsection{Kinetic relations}

In addition to the constitutive assumptions above, we need to prescibe a kinetic relation that governs the propagation of phase boundaries (discontinuities in which the two end states are in different phases). This is necessary in quasistatic situations since the velocity of the phase boundary is indeterminate from the balance laws or equilibrium conditions as we will presently see in Section 3. This is exactly analogous to the situation in bars as pointed out by Ericksen (1975). Similarly in dynamics we shall soon see in Section 4 that unlike classical shocks, the jump conditions alone are not sufficient to uniquely determine the propagation velocity of phase boundaries. This information has to be provided from outside. We do so in the form of a kinetic relation: we assume that the propagation velocity of the phase boundary is a constitutive function of the driving force:

$$
\dot{s}=V(f) .
$$

This notion of a kinetic relation was introduced by Abeyaratne and Knowles (see Abeyaratne and Knowles $(1990,1991)$ ) in their study of bars. We see that the dissipation inequality (19) imposes a restriction on the function $V$ :

$$
f V(f) \geqslant 0 .
$$

For future use, we calculate the driving force for our two-phase material. Substituting (21) and (22) in (18), a long but straightforward calculation shows that the driving force on a phase boundary with the high strain phase on the right is given by

$$
f=E A\left[\left|\alpha^{*}\right|\right]\left(\left\langle\alpha^{*}\right\rangle-\langle\alpha\rangle\right)+\mu A\left[\left|\gamma^{*}\right|\right]\left(\left\langle\gamma^{*}\right\rangle-\langle\gamma\rangle\right)+\Phi_{0} .
$$

Note that we have contributions from the stretch and shear, but none from the curvature. 


\section{Quasistatics}

We now explore the behavior of these beams by studying their equilibrium shapes. We find that the equilibrium shapes are characterized by sharp discontinuities in the tangent and jumps in curvature of the centerline at phase boundaries. We also find that the equilibrium conditions are insufficient to determine the position of the phase boundary, and this provides room for a kinetic relation. We propose a very simple experiment to verify this theory and determine the kinetic relation.

To obtain the equilibrium or quasistatic equations we drop terms associated with inertia in Eqs. (4) and (6) and jump conditions (5) and (7). We obtain

$$
\begin{aligned}
& \mathbf{T}^{\prime}+\mathbf{f}=\mathbf{0}, \quad M^{\prime}+\lambda \hat{\mathbf{t}} \times \mathbf{T}+l=0, \\
& {[|\mathbf{T}|]=\mathbf{0}, \quad[|M|]=0}
\end{aligned}
$$

where $\left({ }^{\prime}\right)$ denotes partial differentiation with respect to $x$. We however allow the phase boundaries to propagate and therefore the kinematic jump conditions (1) and (2) hold unchanged.

\subsection{Beam subjected to pure moments}

Consider a beam subjected to a moment $M$ at its two ends. We assume that there are no forces applied at the ends, and that the body force as well the body moment are zero. Then we see that $\mathbf{T}=\mathbf{0}$ satisfies (26 part 1) and (27 part 1) automatically. Invoking the moment-curvature relation (23), we see that (2), (26 part 2) and (27 part 2) reduce to

$$
\theta^{\prime \prime}=0, \quad\left[\left|\theta^{\prime}\right|\right]=0, \quad[|\theta|]=0,
$$

irrespective of whether the beam is entirely in the low strain phase or in the high strain phase, or whether the beam contains a phase boundary at some point $s$. Clearly the solution is

$$
\theta=\frac{M}{E I} x+c
$$

for some constant $c$. Therefore, the (inherent) curvature in $\kappa=\theta^{\prime}$ is constant through out the beam.

If this beam is entirely in the low strain phase, then we infer from $\mathbf{T}=\mathbf{0}$ and (22) that

$$
\lambda=\lambda_{1}=\sqrt{\alpha_{1}^{2}+\gamma_{1}^{2}}, \quad \beta=\beta_{1}=\arctan \frac{\gamma_{1}}{\alpha_{1}} .
$$

Since $\beta$ is the angle between the tangent and the director, we see that the angle $\zeta$ between the tangent and the horizontal is given by $\zeta=(M / E I) x+c-\beta_{1}$. Recalling that $\mathbf{y}^{\prime}=\lambda \hat{\mathbf{t}}$, we see that

$$
\mathbf{y}=\lambda_{1} \frac{E I}{M}\left(\sin \left(\frac{M}{E I} x+c-\beta_{1}\right) \mathbf{i}-\cos \left(\frac{M}{E I} x+c-\beta_{1}\right) \mathbf{j}\right)+\mathbf{d}
$$

for some constant d. Clearly, the centerline is deformed into a circular arc of radius $(E I / M) \lambda_{1}$.

Similarly if the beam is entirely in the high strain phase, we can follow the arguments above to see that

$$
\mathbf{y}=\lambda_{\mathrm{h}} \frac{E I}{M}\left(\sin \left(\frac{M}{E I} x+c-\beta_{\mathrm{h}}\right) \mathbf{i}-\cos \left(\frac{M}{E I} x+c-\beta_{\mathrm{h}}\right) \mathbf{j}\right)+\mathbf{d}
$$

for some constant $\mathbf{d}$. Clearly, the centerline is deformed into a circular arc of radius $(E I / M) \lambda_{\mathrm{h}}$. 


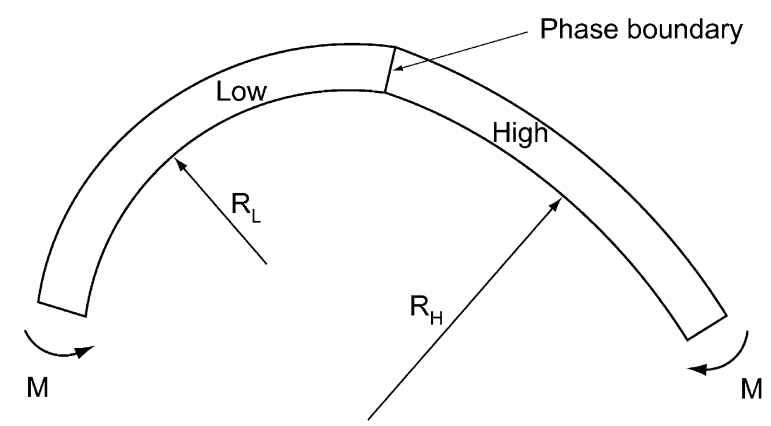

Fig. 2. A beam with a phase boundary subjected to a constant moment.

Now assume that we have a phase boundary at $s$ so that the left part $(0, s)$ of the beam is in the low strain phase while the right part $(s, L)$ of the beam is in the high strain phase. Then arguing as above, we see

$$
\mathbf{y}= \begin{cases}\lambda_{1} \frac{E I}{M}\left(\sin \left(\frac{M}{E I} x+c-\beta_{1}\right) \mathbf{i}-\cos \left(\frac{M}{E I} x+c-\beta_{1}\right) \mathbf{j}\right)+\mathbf{d}^{-} & 0<x \leqslant s, \\ \lambda_{\mathrm{h}} \frac{E I}{M}\left(\sin \left(\frac{M}{E I} x+c-\beta_{\mathrm{h}}\right) \mathbf{i}-\cos \left(\frac{M}{E I} x+c-\beta_{\mathrm{h}}\right) \mathbf{j}\right)+\mathbf{d}^{+} & s<x<L,\end{cases}
$$

for some constants $\mathbf{d}^{-}, \mathbf{d}^{+}$chosen to satisfy $[|\mathbf{y}|]=0$ at $s$. Clearly, the centerline of the left portion of the beam deforms to circular arc of radius $(E I / M) \lambda_{1}$ while the centerline of the right portion deforms to a circular arc of radius $(E I / M) \lambda_{\mathrm{h}}$. This is shown in Fig. 2. While the inherent curvature is uniform, the centerline of the beam - which is the experimentally observable quantity - has two distinct curvatures.

Further, notice that the tangent is discontinuous across the interface (with an angle of $\beta_{\mathrm{h}}-\beta_{1}$ between them). In fact, this angle is independent of the applied moment and is present even at zero moment. Thus, we always expect a kink at the phase boundary.

Finally, we can verify from (25) that the driving force across this interface is exactly equal to $\Phi_{0}$, and thus independent of the applied moment. This means that the propagation of the phase boundary, if any, would be independent of the applied moment.

Berg $(1994,1995)$ designed a clever device to subject polycrystalline wires of NiTi to a pure moment. He observed that the deformed shape of the wire was very much like the predictions above with two distinct curvatures, and modelled it using the Euler-Bernoulli beam theory (which is based on an ansatz of zero stretch and shear) with a non-monotone moment-curvature relation. In other words, he assumed that the energy was convex with infinite modulus in the stretch and shear, while it is non-convex in curvature. This is exactly the opposite of what we have done here. We wonder whether the observations of different curvatures were mediated by different stretch as we have proposed here, or that the polycrystalline wires truly have a non-monotone moment-curvature relation.

There is evidence for the latter in Berg's experiments. First there is no pronounced tangent discontinuity. Second, he could drive the phase boundary by increasing the applied moment in variance with the conclusions above. We note, however, that the phase boundary could have a non-zero driving force even if the moment-curvature relation were convex if the moduli of the two phases were different as in actual materials. It would be very interesting to repeat Berg's experiments with single crystal wires so that these issues can be probed in detail.

\subsection{Cantilever with end load}

Consider a cantilever with the end $x=0$ fixed and with a dead load $-F \hat{\mathbf{j}}$ applied to the end $x=L$ as shown in Fig. 3 (inset). We assume that there are no body forces or moments. The balance of forces 


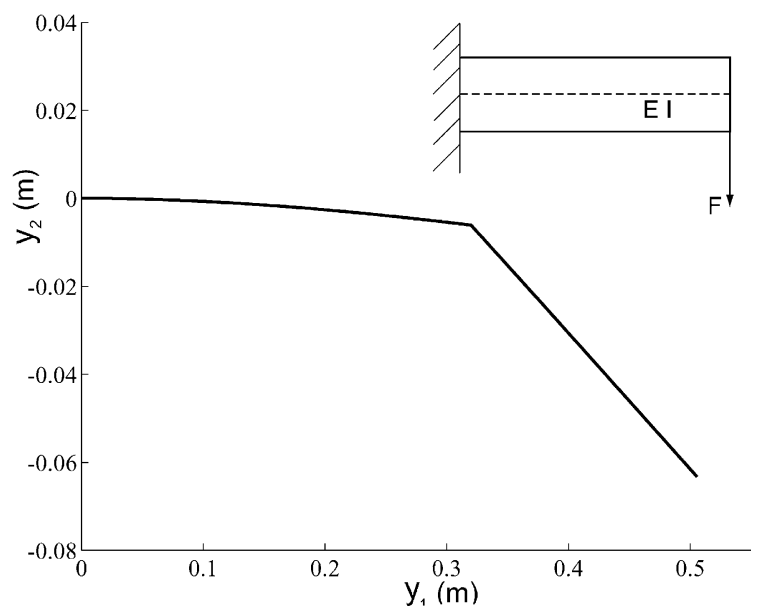

Fig. 3. Typical deformed centerline of a cantilever with a phase boundary subjected to an end load.

((26 part 1) and (27 part 1)) then requires that $\mathbf{T}$ is constant. Therefore, we conclude from the constitutive relation (22) that

$$
\begin{aligned}
& E A\left(\alpha-\alpha^{*}\right) \sin \theta+\mu A\left(\gamma-\gamma^{*}\right) \cos \theta=0, \\
& -E A\left(\alpha-\alpha^{*}\right) \cos \theta+\mu A\left(\gamma-\gamma^{*}\right) \sin \theta=-F .
\end{aligned}
$$

Solving these simultaneously, we obtain

$$
\alpha=\alpha^{*}+\frac{F}{E A} \cos \theta, \quad \gamma=\gamma^{*}-\frac{F}{\mu A} \sin \theta .
$$

In preparation for writing the balance of moments, we use the above to see that

$$
\lambda \hat{\mathbf{t}} \times \mathbf{T}=\left(-\gamma \hat{\mathbf{b}}^{\perp}+\alpha \hat{\mathbf{b}}\right) \cdot(-F \hat{\mathbf{j}})=-F\left(\left(\alpha^{*}+\frac{F}{E A} \cos \theta\right) \sin \theta+\left(\gamma^{*}-\frac{F}{\mu A} \sin \theta\right) \cos \theta\right) .
$$

We use this in the balance of moments (26 part 2) and obtain

$$
E I \theta^{\prime \prime}-F\left(\left(\alpha^{*}+\frac{F}{E A} \cos \theta\right) \sin \theta+\left(\gamma^{*}-\frac{F}{\mu A} \sin \theta\right) \cos \theta\right)=0 .
$$

We have to solve this ordinary differential equation subject to the following boundary conditions if the beam is entirely in one phase:

$$
\theta(0)=\theta_{0}, \quad \theta^{\prime}(L)=0,
$$

and the following boundary and jump conditions if the beam is in two phases

$$
\theta(0)=\theta_{0}, \quad \theta^{\prime}(L)=0, \quad[|\theta|]=0, \quad\left[\left|\theta^{\prime}\right|\right]=0 .
$$

Above, $\theta_{0} \neq 0$ depends on the experimental setup and is fixed for a given setup. In either case (one phase or two phases), this equation can be easily solved numerically by a shooting method. Fig. 3 shows the deformed shape of a beam (centerline) with the following parameters: $E=200.0 \mathrm{GPa}, \mu=80.0 \mathrm{GPa}$, $\alpha_{1}=1.0, \alpha_{\mathrm{h}}=1.043, \gamma_{1}=0.0, \gamma_{\mathrm{h}}=-0.28, L=0.5 \mathrm{~m}, A=0.01 \mathrm{~m}^{2}, I=8.333 \times 10^{-6} \mathrm{~m}^{4}$ and $F=-50.0 \mathrm{~N}$ with a phase boundary at $s=0.3$ separating the low strain phase on the left from the high strain phase on 


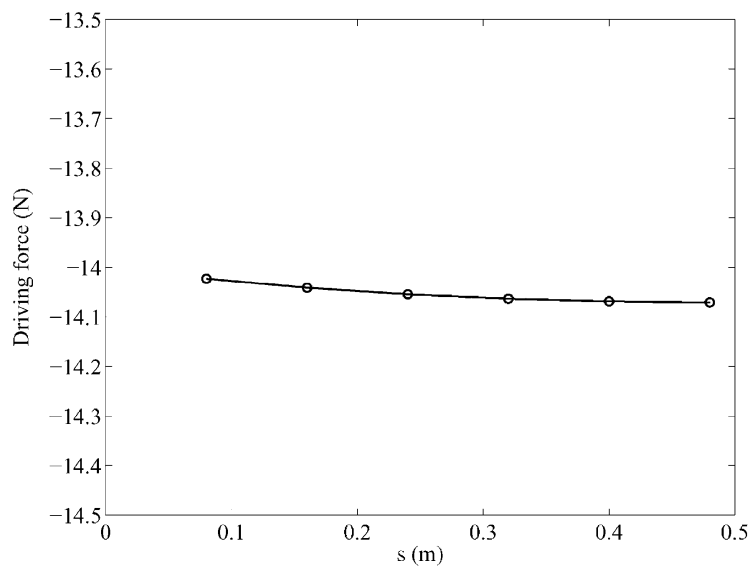

Fig. 4. Driving force on the phase boundary.

the right. Furthermore, unlike the case of pure moments we find that the phase boundary is subjected to a driving force that is linear in $F$. It also depends on the position of the phase boundary but only slightly (as shown in Fig. 4).

This cantilever then provides a simple experiment to determine the kinetic relation. The phase boundary is characterized by a kink and is thus clearly visible. Thus the measurement of the propagation velocity is relatively easy. At the same time we can easily control the driving force through the applied load $F$. Further $\alpha_{\mathrm{h}}, \alpha_{1}, \gamma_{\mathrm{h}}, \gamma_{1}$ can be controlled through sample preparation and $\theta_{0}$ by the clamping condition. This could enable detailed parametric studies.

We now discuss a few limiting cases. If the beam is very slender, (i.e., $I / L^{2} \ll A$ ), then we see that (31) is meaningful if and only if $F=\mathrm{O}\left(E I / L^{2}\right)$, or if $F \ll E A, \mu A$. Therefore, $\alpha=\alpha^{*}, \gamma=\gamma^{*}$, and (31) reduces to

$$
E I \theta^{\prime \prime}-F\left(\alpha^{*} \sin \theta+\gamma^{*} \cos \theta\right)=0 .
$$

We have to solve this subject to the same boundary and jump conditions above.

If on the other hand, the beam is stubby (i.e., if $I / L^{2} \gg A$ ), then $\alpha, \gamma$ become too large to be meaningful unless $F=\mathrm{O}(E A)=\mathrm{O}(\mu A)$. Then, (31) with the appropriate boundary and jump conditions reduces to $\theta=\theta_{0}$. Therefore, (30) yields,

$$
\alpha=\alpha^{*}+\frac{F}{E A} \cos \theta_{0}, \quad \gamma=\gamma^{*}-\frac{F}{\mu A} \sin \theta_{0} .
$$

Suppose, now, that this beam has a phase boundary at point $x=s$ with the low strain phase on the left and the high strain phase on the right. The driving force on this interface is given by (25) as

$$
f=F\left(-\left(\alpha_{\mathrm{h}}-\alpha_{1}\right) \cos \theta_{0}+\left(\gamma_{\mathrm{h}}-\gamma_{1}\right) \sin \theta_{0}\right)+\Phi_{0} .
$$

Therefore, the experiment proposed above would be much simpler in this limit.

Finally, if the beam is stubby, and if the force is small, i.e., if $I / L^{2} \gg A, F / E A \ll \alpha^{*}$ and $F / \mu A \ll \gamma^{*}$ then we have piecewise rigid deformation,

$$
\alpha \approx \alpha^{*}, \quad \beta \approx \beta^{*}, \quad \theta \approx \theta_{0} .
$$

This deformation would be trivial in case the beam was made of a single phase. For example, if the beam was made of the low strain phase, the centerline is straight with an angle $\zeta=\theta_{0}-\beta_{1}$ from the horizontal. 
The deformation is non-trivial in case the beam has a phase boundary at point $x=s$ with the low strain phase on the left and the high strain phase on the right. The centerline would then have a kink at $x=s$ with the left side making an angle $\zeta=\theta_{0}-\beta_{1}$ from the horizontal and the right an angle $\zeta=\theta_{0}-\beta_{\mathrm{h}}$. Further, there is a non-trivial driving force on the phase boundary. The formula (25) cannot be applied here; instead we have to go back to the more fundamental (18). We obtain,

$$
f=\Phi_{0}-(-F \hat{\mathbf{j}}) \cdot\left(\left[\left|\alpha^{*}\right|\right] \hat{\mathbf{b}}^{\perp}+\left[\left|\gamma^{*}\right|\right] \hat{\mathbf{b}}\right)=\Phi_{0}-F\left[\left|\alpha^{*}\right|\right] \cos \theta_{0}+F\left[\left|\gamma^{*}\right|\right] \sin \theta_{0} .
$$

We see that there is a non-trivial driving force on the interface and that this depends on the applied load. James and Rizzoni (in press) and James (in press) have discussed the use of the piecewise rigid approximation in the modelling of active materials.

\section{Shocks and phase boundaries}

We now turn to dynamics and study the restrictions imposed by the jump conditions on the propagation of discontinuities in a two phase material. We call a discontinuity a shock if there is no phase change across it and a phase boundary if there is. We show again that there is room for a kinetic relation.

We begin by combining the kinematic and momentum jump conditions. Eliminating $[|\dot{\mathbf{y}}|]$ between (1) and (5) we obtain,

$$
[|\mathbf{T}|]=\rho A \dot{s}^{2}[|\lambda \hat{\mathbf{t}}|] \text {. }
$$

Substituting for $\mathbf{T}$ from (22), we expand this to be

$$
E A\left(\hat{\mathbf{b}}^{\perp} \otimes \hat{\mathbf{b}}^{\perp}\right)[|\lambda \hat{\mathbf{t}}|]-E A\left[\left|\alpha^{*}\right|\right] \hat{\mathbf{b}}^{\perp}+\mu A(\hat{\mathbf{b}} \otimes \hat{\mathbf{b}})[|\lambda \hat{\mathbf{t}}|]-\mu A\left[\left|\gamma^{*}\right|\right] \hat{\mathbf{b}}=\rho A \dot{s}^{2}[|\lambda \hat{\mathbf{t}}|]
$$

Dividing by $\rho A$ yields,

$$
\left(\dot{s}^{2}-c_{1}^{2} \hat{\mathbf{b}}^{\perp} \otimes \hat{\mathbf{b}}^{\perp}\right)[|\lambda \hat{\mathbf{t}}|]=c_{1}^{2}\left[\left|\alpha^{*}\right|\right] \hat{\mathbf{b}}^{\perp}+c_{2}^{2}(\hat{\mathbf{b}} \otimes \hat{\mathbf{b}})[|\lambda \hat{\mathbf{t}}|]-c_{2}^{2}\left[\left|\gamma^{*}\right|\right] \hat{\mathbf{b}}
$$

where $c_{1}=(E / \rho)^{1 / 2}$ is the bar wave speed and $c_{2}=(\mu / \rho)^{1 / 2}$ is the shear wave speed.

We deal similarly with the terms involving curvature and moment. Eliminating $[|\dot{\theta}|]$ between (2) and (7) we obtain

$$
[|M|]=\rho I \dot{s}^{2}\left[\left|\theta^{\prime}\right|\right]
$$

Substituting for $M$ from (23), and dividing by $\rho I$, we obtain

$$
\left(\dot{s}^{2}-c_{1}^{2}\right)\left[\left|\theta^{\prime}\right|\right]=0 \text {. }
$$

This clearly implies that discontinuities in the curvature $\theta^{\prime}$ necessarily travel at the bar wave speed. This is true irrespective of whether the discontinuity is a shock (in either phase) or a phase boundary. Conversely, a discontinuity travelling at any other speed does not allow the curvature to jump.

There is a far richer class of discontinuities allowed by (32), as we presently see.

\subsection{Shocks}

We first look at discontinuities where both states are in the same phase so that $\left[\left|\alpha^{*}\right|\right]=\left[\left|\gamma^{*}\right|\right]=0$. Substituting these in (32) reduces it to

$$
\dot{s}^{2}[|\lambda \hat{\mathbf{t}}|]=c_{1}^{2}\left(\hat{\mathbf{b}}^{\perp} \otimes \hat{\mathbf{b}}^{\perp}\right)[|\lambda \hat{\mathbf{t}}|]+c_{2}^{2}(\hat{\mathbf{b}} \otimes \hat{\mathbf{b}})[|\lambda \hat{\mathbf{t}}|]
$$


in both the low strain and the high strain phase. Since we may assume without loss of generality that $[|\lambda \hat{\mathbf{t}}|] \neq 0$ (else, we would have no discontinuity), we obtain $\dot{s}^{2}$ and $[|\lambda \hat{\mathbf{t}}|]$ as the eigenvalue and eigenvector of

$$
c_{1}^{2}\left(\hat{\mathbf{b}}^{\perp} \otimes \hat{\mathbf{b}}^{\perp}\right)+c_{2}^{2}(\hat{\mathbf{b}} \otimes \hat{\mathbf{b}}) .
$$

We have two possible solutions,

$$
\dot{s}^{2}=c_{1}^{2} \quad \text { with }[|\lambda \hat{\mathbf{t}}|] \| \hat{\mathbf{b}}^{\perp} \text { and } \dot{s}^{2}=c_{2}^{2} \quad \text { with }[|\lambda \hat{\mathbf{t}}|] \| \hat{\mathbf{b}}
$$

We can thus have shocks which propagate at either the bar or the shear wave speeds, but with constraints in the jump on $\partial \mathbf{y} / \partial x=\lambda \hat{\mathbf{t}}$.

To understand these a little more, let us specialize to the case when the tangent is continuous. Then, the first solutions tells us that arbitrary jumps in the stretch $\lambda$ with no change of phase necessarily have no shear (since the tangent is necessarily perpendicular to the director $\hat{\mathbf{b}}$ ) and travel at the bar wave speed. These are exactly the shock waves that one has in bars. The second solution requires the tangent to be parallel to the director and thus requires an unphysically large amount of shear. Therefore, we believe that this solution is unphysical when the tangent is continuous.

It is interesting to compare these with the case of strings. There, shocks with discontinuous tangent necessarily have no discontinuity in stretch $\lambda$, and propagate at a velocity equal to $(T(\lambda) / \lambda)^{1 / 2}$ (see Purohit and Bhattacharya (in press)). There appears to be no direct analog of these in beams. We wonder if such solutions would appear as limits of certain travelling waves when the bending modulus goes to zero, or if such solutions are dispersive in beams.

\subsection{Phase boundaries}

We now consider discontinuities in which the two end states are in two different phases. We now have to study (32) in all generality. We rewrite it as two scalar equations (components with respect to $\hat{\mathbf{b}}^{\perp}$ and $\hat{\mathbf{b}})$ :

$$
\begin{aligned}
& \left(\dot{s}^{2}-c_{1}^{2}\right)[|\lambda \hat{\mathbf{t}}|] \cdot \hat{\mathbf{b}}^{\perp}=-c_{1}^{2}\left[\left|\alpha^{*}\right|\right], \\
& \left(\dot{s}^{2}-c_{2}^{2}\right)[|\lambda \hat{\mathbf{t}}|] \cdot \hat{\mathbf{b}}=-c_{2}^{2}\left[\left|\gamma^{*}\right|\right] .
\end{aligned}
$$

Clearly these equations do not uniquely determine the velocity of the phase boundary. In fact, it can take a range of values depending on $[|\lambda|]$ and $[|\hat{\mathbf{t}}|]$. It therefore appears that the jump conditions alone are insufficient to determine the phase boundary velocity and that we need to invoke a kinetic relation.

There is an interesting exception, when we a priori know that the tangent is continuous. Then (37) and (38) reduce to

$$
\begin{aligned}
& \left(\dot{s}^{2}-c_{1}^{2}\right)[|\lambda|] \hat{\mathbf{t}} \cdot \hat{\mathbf{b}}^{\perp}=-c_{1}^{2}\left[\left|\alpha^{*}\right|\right], \\
& \left(\dot{s}^{2}-c_{2}^{2}\right)[|\lambda|] \hat{\mathbf{t}} \cdot \hat{\mathbf{b}}=-c_{2}^{2}\left[\left|\gamma^{*}\right|\right] .
\end{aligned}
$$

We can solve these simultaneously for $\dot{s}^{2}$ and $[|\lambda|]$. In particular,

$$
\dot{s}^{2}=c_{1}^{2} c_{2}^{2} \frac{\cos \beta\left[\left|\gamma^{*}\right|\right]-\sin \beta\left[\left|\alpha^{*}\right|\right]}{c_{2}^{2} \cos \beta\left[\left|\gamma^{*}\right|\right]-c_{1}^{2} \sin \beta\left[\left|\alpha^{*}\right|\right]} .
$$

The formulas for $[|\lambda|]$ are omitted for brevity. Thus, the velocity of a phase boundary would be completely determined by the jump conditions if we knew a priori that the tangent is continuous. This appears to be in 
contradiction to the behavior of bars, where the jump conditions do not determine the velocity of the phase boundary. However, a bar is a beam with zero transformation strain in shear $\left[\left|\gamma^{*}\right|\right]=0$ and infinite shear modulus $\mu=\infty$. Taking the limits $\left[\left|\gamma^{*}\right|\right] \rightarrow 0, \mu \rightarrow \infty$ in (40), we see that it reduces to the equation $\hat{\mathbf{t}} \cdot \hat{\mathbf{b}}=0$, and we are now required to find both $\dot{s}^{2}$ and $[|\lambda|]$ from the Eq. (39) which reduces to

$$
\left(\dot{s}^{2}-c_{1}^{2}\right)[|\lambda|]=-c_{1}^{2}\left[\left|\alpha^{*}\right|\right] .
$$

This is exactly the situation in bars. Thus we see that the additional degree of freedom in shear that beams possess, breaks the degeneracy in bars and provides exactly the additional information to determine the phase boundary velocity.

Another noteworthy point here is that when the transformation shear strain $\gamma^{*} \approx 0$ we obtain $\dot{s}^{2} \approx c_{2}^{2}$. This means that phase boundaries with continuous tangent in such materials would travel at nearly the shear wave speed.

\section{Propulsion through a viscous medium}

Various micro-organisms propel through viscous media by beating flagella or cilia. In particular, a flagellum in eucaryotic cells is a slender structure (tens of microns long and tenths of microns wide) that achieves propulsion by actively deforming in a bending wave propagating from one end to the other (see for example Bray (1992)). This deformation is resisted by the viscous motion, and this in turn propels the organism. We saw in the last section that phase boundaries in beams are accompanied by a kink across which one has a large shear. Therefore, by creating and moving the phase boundaries in a particular sequence, the beam can be deformed to mimic these wave like motions.

We assume that the deformation of the beam is piecewise rigid so that $\theta$ is uniform (i.e., $\theta(x, t)=\theta(t)$ ). Consequently the angle that the tangent to the centerline makes with the horizontal is $\zeta(t)+\zeta_{0}$ in the high strain phase and $\zeta(t)-\zeta_{0}$ in the low strain phase. The stretch is $\lambda_{\mathrm{h}}$ in the high strain phase and $\lambda_{1}$ in the low strain phase. We assume that the position and evolution of the phase boundaries is a given input, and try to determine the overall motion of the beam. We do not discuss how one may generate such phase boundaries but refer the reader to Purohit and Bhattacharya (in press). Finally, we only consider quasistatics.

As the beam moves, the viscous medium resists this motion, and we model this resistance as a body force per unit reference length

$$
\mathbf{f}=d_{\mathrm{t}}(\dot{\mathbf{y}} \cdot \hat{\mathbf{t}}) \hat{\mathbf{t}}+d_{\mathrm{n}}(\dot{\mathbf{y}} \cdot \hat{\mathbf{n}}) \hat{\mathbf{n}}
$$

where $d_{\mathrm{t}}$ and $d_{\mathrm{n}}$ are the tangential and normal drag coefficients respectively (see, for instance, Brennen and Winet (1977)).

Suppose we have a beam with two phase boundaries moving periodically in a prescribed manner. Let $s_{1}(t)$ and $s_{2}(t)$ denote the positions of the phase boundaries in the reference configuration, and let the leftmost part of the beam be in the low strain phase. Then given the position $\mathbf{y}_{0}(t)$ of the leftmost point $x=0$, and $\zeta(t)$, we can use the piecewise rigid assumption to write down the position of the centerline as

$$
\mathbf{y}(x, t)= \begin{cases}\mathbf{y}_{0}(t)+\lambda_{1} x \hat{\mathbf{t}}^{-} & 0 \leqslant x \leqslant s_{1}, \\ \mathbf{y}_{0}(t)+\lambda_{1} s_{1} \hat{\mathbf{t}}^{-}+\lambda_{\mathrm{h}}(x-s) \hat{\mathbf{t}}^{+} & s_{1} \leqslant x \leqslant s_{2}, \\ \mathbf{y}_{0}(t)+\lambda_{1} s_{1} \hat{\mathbf{t}}^{-}+\lambda_{\mathrm{h}}\left(s_{2}-s_{1}\right) \hat{\mathbf{t}}^{+}+\lambda_{1}\left(x-s_{2}\right) \hat{\mathbf{t}}^{-} & s_{2} \leqslant x \leqslant L,\end{cases}
$$


where $\hat{\mathbf{t}}^{-}=\cos \left(\zeta-\zeta_{0}\right) \hat{\mathbf{i}}+\sin \left(\zeta-\zeta_{0}\right) \hat{\mathbf{j}}$ and $\hat{\mathbf{t}}^{+}=\cos \left(\zeta+\zeta_{0}\right) \hat{\mathbf{i}}+\sin \left(\zeta+\zeta_{0}\right) \hat{\mathbf{j}}$ respectively. Differentiating with respect to $t$, we obtain the particle velocity

$$
\dot{\mathbf{y}}(x, t)= \begin{cases}\dot{\mathbf{y}}_{0}(t)+\dot{\zeta} \lambda_{1} x \hat{\mathbf{p}}^{-} & 0 \leqslant x \leqslant s_{1}, \\ \dot{\mathbf{y}}_{0}(t)+\lambda_{1} \dot{s}_{1} \hat{\mathbf{t}}^{-}+\dot{\zeta} \lambda_{1} s_{1} \hat{\mathbf{p}}^{-}-\lambda_{\mathrm{h}} \dot{s}_{1} \hat{\mathbf{t}}^{+}+\dot{\zeta} \lambda_{\mathrm{h}}\left(x-s_{1}\right) \hat{\mathbf{p}}^{+} & s_{1} \leqslant x \leqslant s_{2}, \\ \dot{\mathbf{y}}_{0}(t)+\lambda_{1} \dot{s}_{1} \hat{\mathbf{t}}^{-}+\dot{\zeta} \lambda_{1} s_{1} \hat{\mathbf{p}}^{-}+\lambda_{\mathrm{h}}\left(\dot{s_{2}}-\dot{s_{1}}\right) \hat{\mathbf{t}}^{+}+\dot{\zeta} \lambda_{\mathrm{h}}\left(s_{2}-s_{1}\right) \hat{\mathbf{p}}^{+}-\lambda_{1} \dot{s}_{2} \hat{\mathbf{t}}^{-}+\dot{\zeta} \lambda_{1}\left(x-s_{2}\right) \hat{\mathbf{p}}^{-} & s_{2} \leqslant x \leqslant L,\end{cases}
$$

where $\hat{\mathbf{p}}^{-}=-\sin \left(\zeta-\zeta_{0}\right) \hat{\mathbf{i}}+\cos \left(\zeta-\zeta_{0}\right) \hat{\mathbf{j}}$ and $\hat{\mathbf{p}}^{+}=-\sin \left(\zeta+\zeta_{0}\right) \hat{\mathbf{i}}+\cos \left(\zeta+\zeta_{0}\right) \hat{\mathbf{j}}$ are perpendicular to $\hat{\mathbf{t}}^{-}$ and $\hat{\mathbf{t}}^{+}$respectively. We seek to obtain the unknowns $\dot{\mathbf{y}}_{0}(t)$ and $\dot{\zeta}(t)$ using the balance of forces and moments,

$$
\mathbf{0}=\int_{0}^{L}\left\{d_{\mathrm{t}}(\dot{\mathbf{y}} \cdot \hat{\mathbf{t}}) \hat{\mathbf{t}}+d_{\mathrm{n}}(\dot{\mathbf{y}}-(\dot{\mathbf{y}} \cdot \hat{\mathbf{t}}) \hat{\mathbf{t}})\right\} \mathrm{d} x
$$

and

$$
0=\int_{0}^{L}(\mathbf{y}(x)-\mathbf{y}(0)) \times\left\{d_{\mathrm{t}}(\dot{\mathbf{y}} \cdot \hat{\mathbf{t}}) \hat{\mathbf{t}}+d_{\mathrm{n}}(\dot{\mathbf{y}}-(\dot{\mathbf{y}} \cdot \hat{\mathbf{t}}) \hat{\mathbf{t}})\right\} \mathrm{d} x .
$$

A long and cumbersome but unenlightening calculation then yields

$$
\mathbf{A}(\mathbf{s}) \mathbf{R}(\zeta) \mathbf{v}=\mathbf{B}(\mathbf{s}) \dot{\mathbf{s}}
$$

where $\mathbf{A}(\mathbf{s})$ is a $3 \times 3$ matrix, $\mathbf{s}=\left\{s_{1}, s_{2}\right\}^{\mathrm{T}}, \mathbf{R}(\zeta)$ is a $3 \times 3$ rotation matrix of angle $\zeta$ about the axis perpendicular to the plane of the beam, $\mathbf{v}=\left\{\dot{y}_{1}(0, t), \dot{y}_{2}(0, t), \dot{\zeta}(t)\right\}^{\mathrm{T}}, \mathbf{B}(\mathbf{s})$ is a $3 \times 2$ matrix and $\dot{\mathbf{s}}=\left\{\dot{s}_{1}, \dot{s}_{2}\right\}^{\mathrm{T}}$. Assuming that $\mathbf{A}$ is invertible ${ }^{1}$ this equation can be solved to obtain

$$
\mathbf{v}=\mathbf{R}^{\mathrm{T}} \mathbf{A}^{-1} \mathbf{B} \dot{\mathbf{s}} .
$$

Since the phase boundary motion is periodic with period $t$ we integrate this from time 0 to $t$ to see if there is any propulsion. This integration becomes a contour integration on the $\left(s_{1}, s_{2}\right)$-plane. The presence of $\mathbf{R}$ makes it impossible to do the integrations explicitly. The integrations are therefore performed numerically over contours that avoid the singular curve of $\mathbf{A}$. It is possible to integrate the equations even if one chooses a trajectory that crosses (non-tangentially) the singular curve at a finite number of points by requiring the continuity of $\mathbf{y}_{0}$ and $\zeta$. The results from one of these calculations are shown in Fig. 5.

We see that we have significant tumbling, but very little linear motion. While we have not made any systematic attempt to optimize the propulsion by proper choice of trajectory, our numerical experiments suggest that this behavior is typical. The relatively short length of the beam makes it easy to tumble unless the phase boundaries are perfectly symmetric; and any propulsive gains made in one part of the period are reversed in the other part as the phase boundaries reset. It will be interesting to study this in greater detail, perhaps using the framework of geometric phases developed by Kelly and Murray (1995) and Marsden and Ostrowski (1998).

We now propose a strategy to overcome the difficulties above. We show that it is possible to generate linear propulsion with little tumbling by having multiple phase boundaries moving from one end of the beam to the other. Suppose we have a long beam, and we periodically nucleate a phase boundary on the left

\footnotetext{
${ }^{1} \mathbf{A}$ is invertible except on a certain curve in the $\left(s_{1}, s_{2}\right)$-plane. We call this curve the singular curve of $\mathbf{A}$.
} 

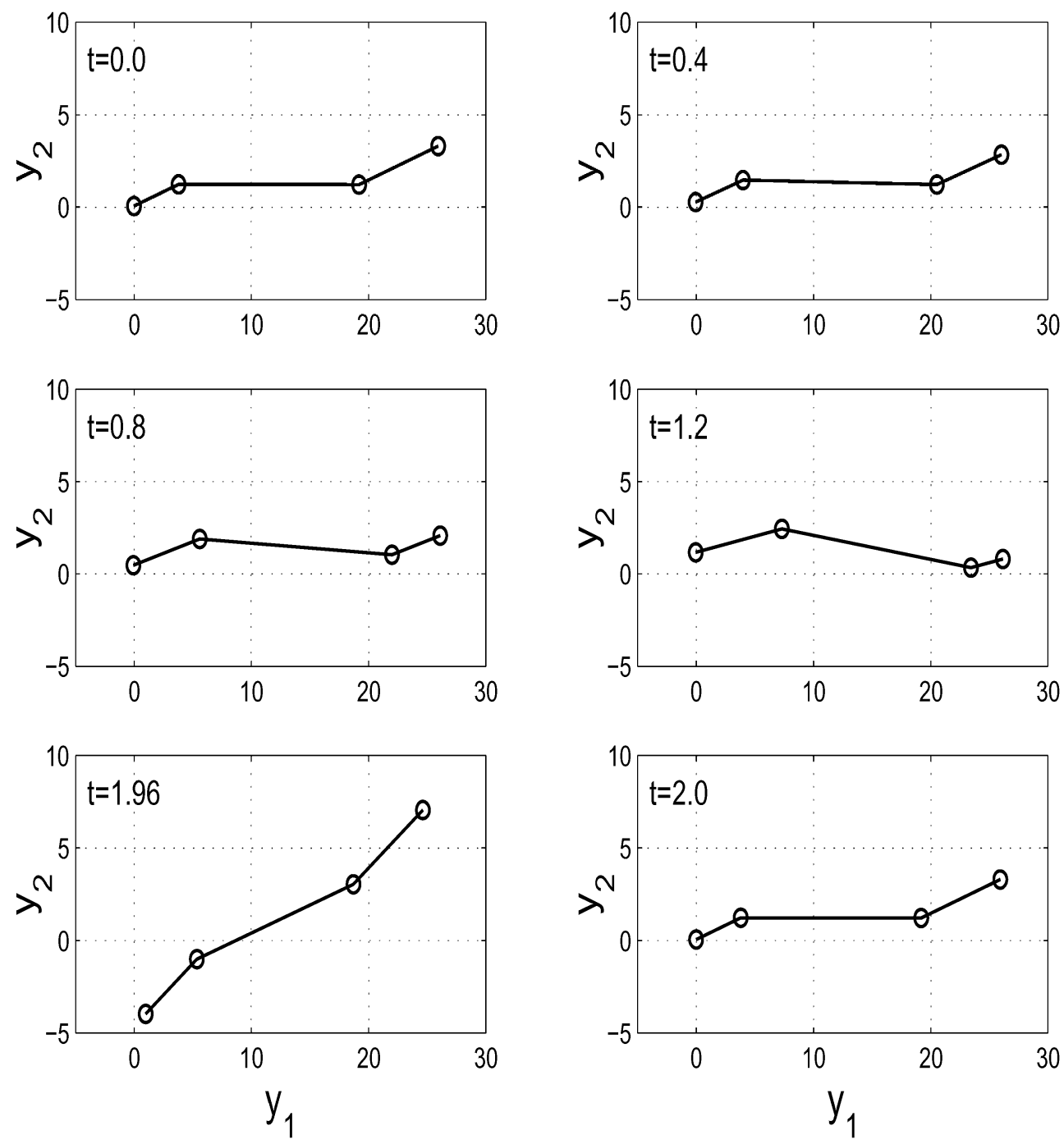

Fig. 5. Positions in space of a beam with two phase boundaries.

$(x=0)$, and have them propagate to the right with a propagation velocity $v$ till they traverse through the entire beam and exit at the right $(x=L)$. Clearly we have to alternately nucleate a low/high and a high/low phase boundary. Then at any instance of time $t$, the beam is composed of alternating segments of the low and high strain phase separated by phase boundaries which have an uniform spacing. This is shown in Fig. 6. Suppose at the instant $t$, the beam has $N$ phase boundaries at the positions $s_{1}(t), s_{2}(t), \ldots, s_{N}(t)$ with

$$
s_{i}-s_{i-1}=d, \quad i=2, \ldots, N .
$$

We refer to the segment of the beam $\left(0, s_{1}\right)$ the first segment, and the segment $\left(s_{i-1}, s_{i}\right)$ to be the $i$ th segment for $i=2, \ldots, N$. 


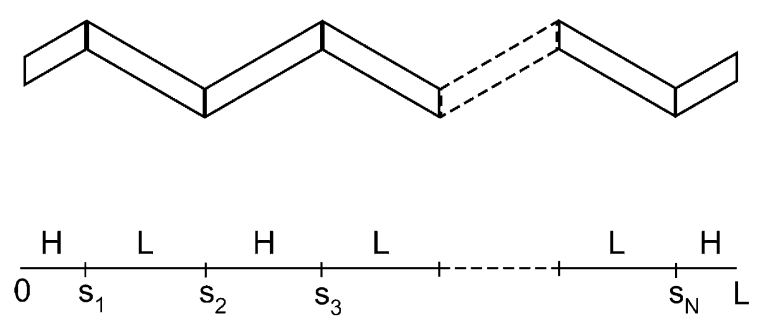

Fig. 6. Beam with multiple phase boundaries.

Let us first assume that the first segment is in the high strain phase; subsequent segments alternate between the low and high strain phases. Therefore,

$$
\lambda(x, t)=\lambda_{i}, \quad \zeta(x, t)=\zeta_{i} \quad s_{i-1}<x<s_{i},
$$

where

$$
\lambda_{i}=\left\{\begin{array}{ll}
\lambda_{\mathrm{h}} & i \text { odd }, \\
\lambda_{1} & i \text { even, }
\end{array} \quad \zeta_{i}= \begin{cases}\zeta_{\mathrm{h}}=\zeta+\zeta_{0} & i \text { odd } \\
\zeta_{1}=\zeta-\zeta_{0} & i \text { even }\end{cases}\right.
$$

The tangent and the normal to the centerline of the beam are

$$
\hat{\mathbf{t}}_{i}=\cos \zeta_{i} \hat{\mathbf{i}}+\sin \zeta_{i} \hat{\mathbf{j}}, \quad \hat{\mathbf{n}}=-\sin \zeta_{i} \hat{\mathbf{i}}+\cos \zeta_{i} \hat{\mathbf{j}}
$$

in the $i$ th segment. Note that $\dot{\hat{\mathbf{t}}}_{i}=\dot{\zeta} \hat{\mathbf{n}}_{i}$. For future use, we set

$$
\begin{aligned}
& \alpha_{i j}=\hat{\mathbf{t}}_{i} \cdot \hat{\mathbf{t}}_{j}=\hat{\mathbf{n}}_{i} \cdot \hat{\mathbf{n}}_{j}= \begin{cases}1 & i+j \text { even, } \\
-\sin 2 \zeta_{0} & i+j \text { odd },\end{cases} \\
& \beta_{i j}=\hat{\mathbf{t}}_{i} \cdot \hat{\mathbf{n}}_{j}=\hat{\mathbf{n}}_{i} \cdot \hat{\mathbf{t}}_{j}= \begin{cases}0 & i+j \text { even, } \\
\sin 2 \zeta_{0} & i+j \text { odd } .\end{cases}
\end{aligned}
$$

The position of the centerline of the beam is given by

$$
\begin{aligned}
\mathbf{y}(x, t) & =\mathbf{y}_{0}(t)+\lambda_{1} s_{1} \hat{\mathbf{t}}_{1}+\sum_{i=2}^{k} \lambda_{i}\left(s_{i}-s_{i-1}\right) \hat{\mathbf{t}}_{i}+\lambda_{k+1}\left(x-s_{k}\right) \hat{\mathbf{t}}_{k+1} \\
& =\mathbf{y}_{0}(t)+\lambda_{1} s_{1} \hat{\mathbf{t}}_{1}+\sum_{i=2}^{k} \lambda_{i} d \hat{\mathbf{t}}_{i}+\lambda_{k+1}\left(x-s_{k}\right) \hat{\mathbf{t}}_{k+1}
\end{aligned}
$$

for $s_{k}<x \leqslant s_{k+1}$. Therefore, the velocity of a point on the centerline is given by

$$
\dot{\mathbf{y}}(x, t)=\dot{\mathbf{y}}_{0}(t)+\dot{\zeta}\left(\lambda_{1} s_{1} \hat{\mathbf{n}}_{1}+\sum_{i=2}^{k} \lambda_{i} d \hat{\mathbf{n}}_{i}+\lambda_{k+1}\left(x-s_{k}\right) \hat{\mathbf{n}}_{k+1}\right)+v\left(\lambda_{1} \hat{\mathbf{t}}_{1}-\lambda_{k+1} \hat{\mathbf{t}}_{k+1}\right)
$$

for $s_{k}<x \leqslant s_{k+1}$.

We first use the balance of angular momentum to show that if $N$ is large, then $\dot{\zeta}=0$, or that the tumbling is eliminated by putting a large number of interfaces. To that end, we calculate 


$$
\begin{aligned}
& l_{f}(x, t)=\left(\mathbf{y}(x, t)-\mathbf{y}_{0}(t)\right) \times \mathbf{f} \\
& =\left(\left(\left(\mathbf{y}-\mathbf{y}_{0}\right) \cdot \hat{\mathbf{t}}\right) \hat{\mathbf{t}}+\left(\left(\mathbf{y}-\mathbf{y}_{0}\right) \cdot \hat{\mathbf{n}}\right) \hat{\mathbf{n}}\right) \times\left(d_{\mathrm{t}}(\dot{\mathbf{y}} \cdot \hat{\mathbf{t}}) \hat{\mathbf{t}}+d_{\mathrm{n}}(\dot{\mathbf{y}} \cdot \hat{\mathbf{n}}) \hat{\mathbf{n}}\right) \\
& =d_{\mathrm{n}}\left(\left(\mathbf{y}-\mathbf{y}_{0}\right) \cdot \hat{\mathbf{t}}\right)(\dot{\mathbf{y}} \cdot \hat{\mathbf{n}})-d_{\mathrm{t}}\left(\left(\mathbf{y}-\mathbf{y}_{0}\right) \cdot \hat{\mathbf{n}}\right)(\dot{\mathbf{y}} \cdot \hat{\mathbf{t}}) \\
& =d_{\mathrm{n}}\left(\lambda_{1} s_{1} \alpha_{1 k+1}+\sum_{i=1}^{k} \lambda_{i} d \alpha_{i k+1}+\lambda_{k+1}\left(x-s_{k}\right)\right) \\
& \left(\dot{\mathbf{y}} \cdot \hat{\mathbf{n}}_{k+1}+\dot{\zeta}\left(\lambda_{1} s_{1} \alpha_{1 k+1}+\sum_{i=1}^{k} \lambda_{i} d \alpha_{i k+1}+\lambda_{k+1}\left(x-s_{k}\right)\right)+v \lambda_{1} \beta_{1 k+1}\right) \\
& -d_{\mathrm{t}}\left(\lambda_{1} s_{1} \beta_{1 k+1}+\sum_{i=1}^{k} \lambda_{i} d \beta_{i k+1}\right) \\
& \left(\dot{\mathbf{y}} \cdot \hat{\mathbf{t}}_{k+1}+\dot{\zeta}\left(\lambda_{1} s_{1} \beta_{1 k+1}+\sum_{i=1}^{k} \lambda_{i} d \beta_{i k+1}\right)+v\left(\lambda_{1} \alpha_{1 k+1}-\lambda_{k+1}\right)\right) \\
& =\dot{\zeta} d^{2}\left(d_{\mathrm{n}}\left(\sum_{i=1}^{k} \lambda_{i} \alpha_{i k+1}\right)^{2}-d_{\mathrm{t}}\left(\sum_{i=1}^{k} \lambda_{i} \beta_{i k+1}\right)^{2}\right) \\
& +d_{\mathrm{n}}\left(\sum_{i=1}^{k} \lambda_{i} d \alpha_{i k+1}\right)\left(a_{k+1}+b_{k+1}\left(x-s_{k}\right)\right)-d_{\mathrm{t}}\left(\sum_{i=1}^{k} \lambda_{i} d \beta_{i k+1}\right) c_{k+1} \\
& +e_{k+1}+f_{k+1}\left(x-s_{k}\right)+g_{k+1}\left(x-s_{k}\right)^{2}
\end{aligned}
$$

for $s_{k}<x \leqslant s_{k+1}$. Therefore,

$$
\begin{aligned}
\int_{s_{k}}^{s_{k+1}} l_{f} \mathrm{~d} x= & \dot{\zeta} d^{3}\left(d_{\mathrm{n}}\left(\sum_{i=1}^{k} \lambda_{i} \alpha_{i k+1}\right)^{2}-d_{\mathrm{t}}\left(\sum_{i=1}^{k} \lambda_{i} \beta_{i k+1}\right)^{2}\right) \\
& +d_{\mathrm{n}}\left(\sum_{i=1}^{k} \lambda_{i} d \alpha_{i k+1}\right)\left(a_{k+1} d+b_{k+1} \frac{d^{2}}{2}\right)-d_{\mathrm{t}} d\left(\sum_{i=1}^{k} \lambda_{i} d \beta_{i k+1}\right) c_{k+1} \\
& +d e_{k+1}+f_{k+1} \frac{d^{2}}{2}+g_{k+1} \frac{d^{3}}{3} .
\end{aligned}
$$

Now note that

$$
\lambda_{i} \alpha_{i k+1}= \begin{cases}\lambda_{\mathrm{h}} & i \text { odd } k+1 \text { odd } \\ -\lambda_{\mathrm{h}} \sin 2 \zeta_{0} & i \text { odd } k+1 \text { even } \\ -\lambda_{1} \sin 2 \zeta_{0} & i \text { even } k+1 \text { odd } \\ \lambda_{1} & i \text { even } k+1 \text { even }\end{cases}
$$

so that

$$
\sum_{i=1}^{k} \lambda_{i} \alpha_{i k+1} \approx \frac{k}{4}\left(\lambda_{\mathrm{h}}\left(1-\sin 2 \zeta_{0}\right)+\lambda_{1}\left(1-\sin 2 \zeta_{0}\right)\right)
$$


for $k$ large enough and averaged over $k$ odd and even. Similarly,

$$
\sum_{i=1}^{k} \lambda_{i} \beta_{i k+1} \approx \frac{k}{4}\left(\lambda_{\mathrm{h}} \sin 2 \zeta_{0}+\lambda_{1} \sin 2 \zeta_{0}\right)
$$

for $k$ large enough and averaged over $k$ odd and even. Substituting these in (50), we see that

$$
\int_{s_{k}}^{s_{k+1}} l_{f} \mathrm{~d} x=\frac{\dot{\zeta} d^{3}\left\langle\lambda^{*}\right\rangle}{16} k^{2}\left(d_{\mathrm{n}}\left(1-2 \sin 2 \zeta_{0}+\sin ^{2} 2 \zeta_{0}\right)-d_{\mathrm{t}} \sin ^{2} 2 \zeta_{0}\right)+\mathrm{O}(k)
$$

Therefore, if the number of phase boundaries $N$ is large enough,

$$
\int_{0}^{L} l_{f} \mathrm{~d} x \approx \sum_{k=1}^{N} \int_{s_{k}}^{s_{k+1}} l_{f} \mathrm{~d} x \approx \frac{\dot{\zeta} d^{3} N^{3}\left\langle\lambda^{*}\right\rangle}{48}\left(d_{\mathrm{n}}\left(1-2 \sin 2 \zeta_{0}+\sin ^{2} 2 \zeta_{0}\right)-d_{\mathrm{t}} \sin ^{2} 2 \zeta_{0}\right)+\mathrm{O}\left(N^{2}\right) .
$$

Therefore we see that if $N$ is large enough, the balance of angular momentum,

$$
\int_{0}^{L} l_{f} \mathrm{~d} x=0
$$

implies that

$$
\dot{\zeta}=0 \text {. }
$$

Now, according to (41) and (49), the body force per unit length,

$$
\mathbf{f}=d_{\mathrm{t}}\left(\dot{\mathbf{y}}_{0} \cdot \hat{\mathbf{t}}_{k+1}\right) \hat{\mathbf{t}}_{k+1}+d_{\mathrm{n}}\left(\dot{\mathbf{y}}_{0} \cdot \hat{\mathbf{n}}_{k+1}\right) \hat{\mathbf{n}}_{k+1}+v\left(d_{\mathrm{t}}\left(\lambda_{1} \alpha_{1 k+1}-\lambda_{k+1}\right) \hat{\mathbf{t}}_{k+1}+d_{\mathrm{n}} \lambda_{1} \beta_{1 k+1} \hat{\mathbf{n}}_{k+1}\right)
$$

for $s_{k}<x \leqslant s_{k+1}$. Therefore for $N$ large enough,

$$
\int_{0}^{L} \mathbf{f} \mathrm{d} x=\sum_{k=1}^{N} \int_{s_{k}}^{s_{k+1}} \mathbf{f} \mathrm{d} x \approx \mathbf{A} \dot{\mathbf{y}}-v \mathbf{a}_{\mathrm{h}},
$$

where

$$
\begin{aligned}
& \mathbf{A}=d_{\mathrm{t}}\langle\hat{\mathbf{t}} \otimes \hat{\mathbf{t}}\rangle+d_{\mathrm{n}}\langle\hat{\mathbf{n}} \otimes \hat{\mathbf{n}}\rangle, \\
& \mathbf{a}_{\mathrm{h}}=\frac{1}{2}\left(d_{\mathrm{t}}\left(\lambda_{\mathrm{h}} \sin 2 \zeta_{0}+\lambda_{1}\right) \hat{\mathbf{t}}_{\mathrm{l}}-d_{\mathrm{n}} \lambda_{\mathrm{h}} \sin 2 \zeta_{0} \hat{\mathbf{n}}_{\mathrm{l}}\right) .
\end{aligned}
$$

Therefore, the balance of forces gives us

$$
\dot{\mathbf{y}}=v \mathbf{A}^{-1} \mathbf{a}_{\mathrm{h}} \text {. }
$$

This gives the velocity as long as the first segment is in the high strain phase.

We can do a similar calculation when the first segment is in the low strain phase. We obtain

$$
\dot{\mathbf{y}}=v \mathbf{A}^{-1} \mathbf{a}_{1},
$$

where

$$
\mathbf{a}_{1}=\frac{1}{2}\left(d_{\mathrm{t}}\left(\lambda_{1} \sin 2 \zeta_{0}+\lambda_{\mathrm{h}}\right) \hat{\mathbf{t}}_{\mathrm{h}}-d_{\mathrm{n}} \lambda_{1} \sin 2 \zeta_{0} \hat{\mathbf{n}}_{\mathrm{h}}\right)
$$

Therefore, we obtain a propulsion with overall velocity

$$
\frac{v}{2} \mathbf{A}^{-1}\left(\mathbf{a}_{\mathrm{h}}+\mathbf{a}_{1}\right) \text {. }
$$


Two aspects were crucial above. First, that the beam was long with a large number of interfaces. This seems important for preventing tumbling. Second, all the phase boundaries moved in one direction. This is consistent with the behavior observed in flagella.

\section{Acknowledgements}

We gratefully acknowledge the partial financial support of the Air Force Office of Scientific Research through a MURI grant (F 49620-98-1-0433).

\section{References}

Abeyaratne, R., Knowles, J.K., 1990. On the driving traction on a surface of a strain discontinuity in a continuum. J. Mech. Phys. Solids 38,345 .

Abeyaratne, R., Knowles, J.K., 1991. Kinetic relations the propagation of phase boundaries in solids. Arch. Rational Mech. Anal. 114, 119.

Antman, S.S., 1995. Non-linear problems of elasticity. Springer-Verlag, New York.

Ball, J.M., James, R.D., 1992. Proposed experimental tests of a theory of fine microstructure and the two-well problem. Phil. Trans. Royal Soc. Lond. A 338, 389-450.

Berg, B.T., 1994. Thermomechanics of shape memory alloy rods, Ph.D Thesis, University of Minnesota.

Berg, B.T., 1995. Bending of superelastic wires 1-experimental aspects. J. Appl. Mech. 62 (2), 459-465.

Bhattacharya, K., 1991. Wedge-like micro-structure in martensites. Acta Metall. Mater. 39, 2431-2444.

Bhattacharya, K., in press. A direct derivation of a theory of rods with application to shape-memory alloys.

Bhattacharya, K., James, R.D., 1999. A theory of thin films of martensitic materials with applications to microactuators. J. Mech. Phys. Solids 47, 531-576.

Bray, D., 1992. Cell Movements. Garland Publishing, New York.

Brennen, C., Winet, H., 1977. Fluid mechanics of propulsion by cilia and flagella. Ann. Rev. Fluid Mech. 9, 339-398.

Coleman, B.D., Noll, W., 1963. The thermodynamics of elastic materials with heat conduction and viscosity. Arch. Rational Mech. Anal. 13, 167-178.

Ericksen, J.L., 1975. Equilibrium of bars. J. Elasticity 5, 191.

Escobar, J., Clifton, R., 1993. On pressure-shear plate impact for studying the kinetics of stress-induces phase transitions. Mater. Sci. Eng. A $170,125-142$.

Eshelby, J.D., 1956. In: Solid State Physics, vol. 3. Academic press, New York, pp. 79-144.

Eshelby, J.D., 1975. The elastic energy-momentum tensor. J. Elasticity 5, 321.

James, R.D., in press. I. Real and configurational forces in magnetism, and II. Analysis of a microscale cantilever. Continuum Mech. and Thermodynamics (issue in honor of Ingo Müller).

James, R.D., Rizzoni, R., in press. Piecewise rigid body mechanics.

Kelly, S.D., Murray, R.M., 1995. Geometric phases and robotic locomotion. J. Robotic Syst. 12 (6), 417-431.

Marsden, J.E., Ostrowski, J., 1998. Symmetries in motion: Geometric foundations of motion control. Nonlinear Sci. Today.

Purohit, P.K., 2001. Dynamics of phase transitions in strings and beams, Ph.D Thesis, California Institute of Technology.

Purohit, P.K., Bhattacharya, K., in press. Dynamics of strings made of a phase transforming material.

Truskinovsky, L., 1987. Dynamics of nonequilibrium phase boundaries in a heat conducting non-linear elastic medium. J. Appl. Math. Mech. (PMM) 51, 777-784. 